\title{
Application of motivational interviewing to prenatal smoking cessation: training and implementation issues
}

Mary M Velasquez, Jacklyn Hecht, Virginia P Quinn, Karen M Emmons, Carlo C DiClemente, Patricia Dolan-Mullen

Department of Family Practice and

Community Medicine,

University of

Texas-Houston

Medical School,

Houston, Texas, USA

M M Velasquez

Center for Behavioral and Preventive

Medicine, Miriam

Hospital

J Hecht

Kaiser Permanente Southern California, Pasadena, California, USA

V P Quinn

Dana Farber Cancer Institute and Harvard School of Public

Health, Boston,

Massachusetts, USA

K Emmons

Department of

Psychology, University

of

Maryland-Baltimore

County, Baltimore,

Maryland, USA

C C DiClemente

Center for Health Promotion Research and Prevention, University of

Texas-Houston Health Science Center, School of Public Health

P Dolan-Mullen

Correspondence to: Mary M Velasquez, $\mathrm{PhD}$, Department of Family

Practice and Community

Medicine, University of

Texas-Houston Medical

School, 6431 Fannin, Suite

2.112, Houston, TX

77030-1501, USA:

mvelasquez@fpcm.med.uth.

tmc.edu

Received 8 December 1999 and in revised form

27 March 2000

Accepted 5 April 2000

\section{Abstract}

Objective-Three of the Smoke-Free Families projects incorporated motivational interviewing (MI) into prenatal smoking cessation interventions. This paper describes the process involved in training healthcare providers to use MI and the issues encountered in implementing the protocols.

Design-Health care providers at all three sites attended local training workshops in which they learned to apply the basics of MI to their study protocol. All sites followed a similar outline and schedule for training and monitoring.

Settings-The MI interventions were delivered through home visits in Boston, Massachusetts; phone based counselling calls to patients' homes in Southern California; and in urban and rural prenatal clinics throughout East Texas.

Participants-Public health nurse and social work case managers, who were already employed by health care agencies, delivered the MI interventions.

Measures-Pre- and postintervention assessments and feedback from trainers and investigators at all three sites.

Results-Providers were enthusiastic about the training workshops, which they rated as effective in preparing them to deliver the intervention. Barriers to implementation included difficulty in contacting patients and competing demands on providers' time.

Conclusions-Conducting initial training for providers is the first step in developing skills to deliver motivational interventions. Additional time and resources are needed for ongoing skill building and monitoring of intervention delivery.

(Tobacco Control 2000;9(Suppl III):iii36-iii40)

Keywords: pregnancy; smoking; motivational interviewing
In response to the Robert Wood Johnson Foundation's call for proposals for the SmokeFree Families (SFF) initiative, three sites proposed to use a motivational interviewing (MI) approach in the delivery of their interventions. The shared goal of all three sites was to test the effectiveness of a brief motivational intervention delivered by nurses, social workers or case managers who provide prenatal health care. A motivational approach has shown promise as a means of facilitating change in settings such as health care agencies, where clinicians' exchanges with patients are relatively brief. ${ }^{12}$ At an early investigator's meeting, grantees from Dana Farber Cancer Institute (DFCI) in Boston, Massachusetts, Kaiser Permanente Southern California (KPSC), and the University of Texas-Houston School of Public Health (UT-H) discovered the similarities in their intervention designs and discussed the advantages and feasibility of coordinating training efforts. Collaboration was facilitated by four members of the investigative teams with extensive experience in training practitioners to use MI. It was thought that by using the same trainers (or configurations thereof) to conduct training at all three sites, the three studies would benefit from an important degree of standardisation.

The similarities and differences between study sites are presented in table 1 . While nurses and/or social workers from participating agencies were trained to deliver the interventions at all three sites, the health care settings and primary work responsibilities differed. The target participants also differed in a number of ways, although all were pregnant smokers. For example, Dana Farber's Healthy Baby Program (HBP) trained public health nurses to deliver the intervention through in-home visits to low income women who lived in inner city and suburban areas in and around the Boston metropolitan area. The KPSC study used nurse case managers who worked in a regionwide preterm prevention

Table 1 Comparison of the three SSF sites using motivational interviewing

\begin{tabular}{|c|c|c|c|c|}
\hline Site & Geographic location & Health care setting & Intervention providers & Target population \\
\hline $\begin{array}{l}\text { Dana Farber Cancer Institute } \\
\text { Healthy Babies Program }\end{array}$ & $\begin{array}{l}\text { Inner city and surrounding areas } \\
\text { in Boston, Massachusetts }\end{array}$ & $\begin{array}{l}\text { State funded, high risk pregnancy } \\
\text { home visitation program }\end{array}$ & $\begin{array}{l}\text { Public health nurses and } \\
\text { case managers }\end{array}$ & $\begin{array}{l}\text { High risk, low income } \\
\text { pregnant smokers }\end{array}$ \\
\hline $\begin{array}{l}\text { University of Texas-Houston } \\
\text { Pregnancy, Stress and Smoking } \\
\text { Study }\end{array}$ & $\begin{array}{l}\text { Houston, Texas and surrounding } \\
\text { urban and rural areas }\end{array}$ & $\begin{array}{l}10 \text { urban and rural medical } \\
\text { school based perinatal care } \\
\text { clinics }\end{array}$ & $\begin{array}{l}\text { Nurse and social worker } \\
\text { case managers }\end{array}$ & $\begin{array}{l}\text { High risk, low income } \\
\text { pregnant smokers }\end{array}$ \\
\hline $\begin{array}{l}\text { Kaiser Permanente Southern } \\
\text { California }\end{array}$ & Southern California & $\begin{array}{l}\text { Large group model health } \\
\text { maintenance organisation }\end{array}$ & Nurse educators & Pregnant smokers \\
\hline
\end{tabular}


program that monitored patients through phone based counselling. The UT-H providers were nurse or social worker case managers employed in 10 urban and rural prenatal care clinics throughout East Texas. Thus, at all three sites, the providers who delivered the intervention were employed by a participating health care agency, and were not hired exclusively to deliver the smoking intervention. At the DCFI and UT-H sites the providers were responsible for helping their patients with numerous other clinical and social problems beyond delivering the smoking intervention; at KPSC the nurse educators had clinical responsibilities with other patients, in addition to counselling enrolled smokers.

\section{Description of $M I$ and rationale for use in SFF studies}

Motivational interviewing is a directive, patient centred counselling style that emerged as an effective approach for helping people with problem drinking. ${ }^{3}$ Since its development, MI has been tested and adapted for brief encounters in medical and other settings. ${ }^{4}{ }^{5}$ The MI counselling approach is based on the assumption that the responsibility and capability for change lies within the patient. The counsellor's task is to create a set of conditions that will enhance the patient's motivation for and commitment to change. This is done by helping people work through their ambivalence about changing, primarily through five basic strategies: (1) Asking open ended questions; (2) reflective (active) listening; (3) developing discrepancy; (4) providing personalised feedback; and (5) eliciting self motivating statements. Reflective listening is a process of hearing what the patient says and gently assisting her to elaborate and clarify her feelings further. Developing discrepancy helps the patient to recognise and compare both the "good" and "not so good" aspects of her smoking, and is an important strategy that can help to motivate patients towards change. When these strategies are implemented effectively, they often prompt patients to make self motivating statements that suggest change is possible. These techniques are described as the "microskills" that form the foundation of MI; when used together they encourage the patient to clarify her perspective and facilitate change. Providing personalised feedback helps patients to recognise the links between a behaviour, such as smoking, and the personal consequences they experience or anticipate.

Health care organisations that support ongoing relationships between providers and patients appear to be ideal settings for incorporating the empathic counselling style of $\mathrm{MI}$ to assist patients with health behaviour change. The SFF initiative presented an opportunity to test and compare the feasibility of training nurses, case managers, and social workers to incorporate a motivational intervention for smoking into the ongoing care they provide to high risk, pregnant women.

\section{Training methods and results}

There were four main trainers who, in collaboration with the principal investigators, facilitated the training workshops; at least two of these trainers conducted the workshops at each site. The workshops were conducted in either a one or two day format to best accommodate the sites' scheduling needs. The content of the MI training was the same across the three sites, although the particular applications varied according to the protocols. Follow up training and monitoring of implementation was planned individually for each site. This section describes the training for each of the sites.

DANA FARBER CANCER INSTITUTE

Training for the Healthy Baby Second-Hand Smoke Study consisted of an eight hour, one day workshop. Two training workshops with the same content and format were conducted a week apart to enable all providers to attend and receive individualised feedback on ways to refine their motivational skills. In total, 23 nurses attended the workshop, which was designed to build upon successful strategies that nurses were already using to help their patients solve difficult life and health problems. A primary goal of the training was to reinforce and enhance motivational skills through a combination of didactic and experiential learning. The morning session engaged all of the nurses in practicing the basic MI; in the afternoon the nurses incorporated these skills into their practice of the research protocol. The study protocol is described in detail by Emmons and colleagues. ${ }^{7}$

The training began with introductions and a discussion of the difficulties nurses encounter when trying to help patients with health behaviour change. The trainers reviewed theories of health behaviour change and basic principles and strategies of MI. The trainers then demonstrated and facilitated role play exercises of these motivational skills. Efforts to enhance development of motivational skills included demonstrating and having nurses practice the five basic MI strategies that were described earlier. After practicing these skills individually, the nurses were coached in how to incorporate these skills into their practice of the standardised research protocol. Special emphasis was also placed on how to present feedback on the participant's household nicotine concentrations. The workshop concluded with a discussion about additional training and resources the nurses might need and having them complete a written evaluation of the training workshop.

Overall, the nurses found the training to be very useful and rated the quality of the training quite high on the post-training questionnaires. In particular, they reported appreciating the potential impact of doing more listening and less direct advice giving, and commented that they learned new strategies to help them address other health behaviours that were of concern to their patients. They reported moderate to high confidence in having learned the basic MI skills to deliver the research protocol. Additional needs that nurses identified included having more information about effective strategies for smoking cessation and 
additional practice time to review the research protocol. They also wanted more guidance on determining how to give the corresponding patient education handouts, and how to complete the process evaluation tools. The process evaluation tool was a brief checklist to document the extent to which the nurse delivered the core intervention components and how much time she spent talking about smoking with each patient.

The research team provided additional training, practice, and educational materials in response to feedback from the initial training. For example, the nurses participated in an additional workshop to review effective strategies for smoking cessation, which was based on principles of adult learning, behaviour change theory, and the Agency for Health Care Policy and Research guidelines for smoking cessation. The training team also conducted one-on-one role plays with each provider who enrolled participants to enhance the quality of intervention delivery. Furthermore, the research team developed additional worksheets for the nurses to use interactively with their patients to help them think about new coping strategies and social support that might make behaviour change more successful. While the trainers had planned to make home visits with the nurses to observe intervention delivery in the field and maximise treatment fidelity, spontaneous scheduling changes and the frequency of patients not being home when the nurse arrived made this level of monitoring difficult to accomplish. Ongoing follow up and supervision was therefore limited to meeting nurses at their agency, during their scheduled documentation time, to review cases and practice ways of handling difficult situations.

UNIVERSITY OF TEXAS-HOUSTON

The UT-H Pregnancy, Stress and Smoking Study training consisted of a two day workshop held in Houston, Texas. A workshop with the same format and content was held two weeks later for those providers who were unable to attend the first training. A total of 20 case managers attended the workshops.

Each 14 hour training workshop was designed to teach the providers the basic constructs of the transtheoretical model (TTM) and MI. Like the DFCI workshop, the training was both didactic and experiential. The case managers were taught the background and history of the TTM and MI, and they were assisted in learning the skills through a series of demonstrations and role plays. On the morning of the first day, training focused on the stages and processes of change and the delivery of a personalised feedback report based on these variables. In the afternoon the case managers were taught the basic principles of MI. This training was similar to that conducted at the DFCI training and described in detail above. An overview of the study protocol was provided toward the end of day 1 (see Mullen and colleagues, this issue, for a description of the intervention ${ }^{8}$ ).

The second day of training was devoted to describing the study protocol in detail and integrating $\mathrm{MI}$ strategies into the delivery of the feedback, decisional balance, and change plan development. In evaluating the training session, case managers were asked to rate their level of achievement for meeting the objectives of the training. On a five point Likert scale with $1=$ "I learned nothing", and $5=$ "I learned a great deal", mean ratings were as follows: participants rated their ability to identify the characteristics of patients in each stage of change as 3.6; ability to integrate fundamental MI skills into their own case management style as 3.5; ability to integrate $\mathrm{MI}$ into the study protocol as 3.4; and confidence in using $\mathrm{MI}$ as 3.3. Trainees reported finding the training to be "interesting and informative", and many expressed feeling that they would be able to incorporate $\mathrm{MI}$ in all areas of their practice.

Plans for follow up training consisted of a study supervisor travelling to the clinics to observe at least one session per case manager. Following the session, the supervisor provided feedback on the case manager's use of MI and adherence to the study protocol. Case managers were also taught to monitor their own delivery of the intervention using a process evaluation worksheet on which they could verify that each component of the intervention was delivered. Quarterly meetings were also scheduled to allow supervisors to work with case managers to refine their skills and problem solve any issues in protocol delivery. Follow up training and monitoring often fell short of the ideal, however, owing to competing demands on providers' time.

KAISER PERMANENTE SOUTHERN CALIFORNIA

The KPSC training consisted of a six hour workshop that included lectures on the TTM and $\mathrm{MI}$ and their application to prenatal smoking behaviour change; 33 providers attended this workshop. The training also included practice in assessing stage of change and administering decisional balance exercises as well as viewing a videotaped staged MI session between a counsellor and pregnant smoker. As in the DFCI and UT-H studies, trainers demonstrated MI strategies and "coached" providers as they used role plays to practise the various techniques. Trainees described the training as effective in preparing them to implement the intervention (mean 4.4 on a scale from 1 to 5 ) and reported that it improved their ability to counsel patients (mean of 4.0). They also expressed a desire for additional training sessions (mean of 3.9).

Within two months of the initial session, the counsellors attended a two hour, small group meeting. This meeting reviewed the principles and strategies of $\mathrm{MI}$ and introduced the research protocols. In response to requests from workshop attendees, the follow up meeting included an overview of cognitive/ behavioural strategies commonly used in smoking interventions. The prenatal educators were given an 86 page reference manual and KPSC provided eight hours of educational time for reading and review. 
IMPLEMENTATION ISSUES

In sum, while there were both similarities and differences in their protocols, all three sites depended on providers' use of MI as a means of delivering the various components of the interventions. Post-training evaluations indicated that the trainees felt at least moderately confident in their abilities to use MI strategies to deliver the intervention protocols. They also expressed a need for ongoing training and case supervision. The investigators all agreed that the initial training was only a "start" and each site made its own provisions for supervision and monitoring of the intervention delivery.

Each site encountered challenges in protocol implementation. While some of the issues were specific to each site, other barriers were common to all.

At DFCI, the primary challenges in implementing the intervention within the public health nursing system in the HBP related to the difficulty in contacting study participants. Lack of working telephones and the transient nature of the population often resulted in patients not being available for nursing visits. Limited resources precluded having the providers increase their number of contact attempts to complete the study intervention. The end result was that many of the study patients received less than the optimal or planned dose of the intervention. Furthermore, these difficulties in scheduling home visits required frequent, unplanned drop-in visits, which made it hard to provide the field supervision of intervention delivery.

At UT-H, shortly after the start of the trial, the site encountered some problems that would greatly affect implementation. As described by Mullen and colleagues, ${ }^{4}$ a number of small rural clinics were added to the previously existing clinic network, which greatly extended the caseloads and duties of the study providers. This resulted in high staff turnover and a decrease in morale. A number of patients also left the clinics during this time because of changes in Medicaid policies and the resulting ability for patients to obtain private care. Providers, who were already quite strapped for time, were often reluctant to set appointments for study supervisors to monitor delivery of the study protocol and their use of MI. In the five cases in which supervisors were able to observe actual sessions, the degree to which providers actually used MI was quite limited. While a few providers demonstrated good skills and some mastery of the protocol, these providers often reported that the observed session was "unusual" and due in large part to the fact that they had taken time to prepare in advance since they were going to be observed. Providers reported that they often did not even have the few extra minutes it took to deliver a woman's personalised feedback or to complete a decisional balance exercise, and that they were generally unable to review and practice the MI techniques they had learned in training. Busy clinics were often unable to provide "release time" for providers to attend follow up training sessions, so two group (45 minute) conference calls were held in lieu of the planned quarterly meetings. Overall, in spite of great effort on the part of the study staff, and good intentions on the parts of the providers, the actual delivery of the intervention was quite limited.

While the KPSC site encountered many of the same difficulties, they were the most successful of the three sites in actual delivery of the intervention protocol. As described by Ershoff and colleagues, ${ }^{9}$ counsellors were able to reach $79 \%(n=80)$ of the patients in the MI group, and patients received on average four calls each of 12 minutes duration. Even in this setting, however, in which the providers were employed by the grantee agency, they reported that a number of factors impeded the full delivery of the intervention. Among difficulties reported on a 1 to 5 scale of importance (where $1=$ "not at all" and $5=$ "very"), were "not enough time" (mean 3.8), "competing priorities" (mean 4.0), and "difficulties reaching patients by telephone" (mean 4.4). Further, nurses found that it was often necessary to make proactive telephone calls beyond usual working hours in order to reach patients. This placed a considerable burden on the counsellors and had a negative impact on their motivation.

The KPSC providers reported higher success in contacting patients and in delivering the components of the protocol. However, it is not known to what extent they actually used MI in their sessions. Although a study investigator contacted all providers via telephone at least once during the study, and was available for problem solving, funding limitations precluded in-person supervision sessions or monitoring of calls.

\section{Discussion}

There were a number of lessons learned from the implementation of these three studies. Some of the challenges related to limited resources, while others were a function of organisational and/or contextual factors.

MASTERY OF MOTIVATIONAL INTERVIEWING SKILLS

Teaching providers new skills and helping them to incorporate new techniques into routine practice is a process that takes time. The full impact of the skills that nurses acquired from the training and their field experience may not have been fully captured within the study period. There seemed to be a cohort of providers who recognised the value of enhancing smoking interventions, and who could more easily incorporate the style of MI soon after the initial training. Other providers never fully embraced the intervention and ultimately had little or no adherence to the study protocol and motivational style of counselling. Rather than training all providers, it may be more (cost) effective to identify a select group who are interested and can most easily learn and apply new counselling skills when evaluating new behaviour change interventions.

LIMITED FOLLOW UP TRAINING AND MONITORING Conducting an initial training for providers is just the first step in helping them develop and refine the skills necessary to deliver a truly motivational intervention. As these studies 
were designed to assess feasibility, and not as clinical (efficacy) trials, they were limited in budget and scope. Although the majority of providers described their training as effective in preparing them to implement the intervention, it is unlikely that providers at any of the sites were able to incorporate the skills consistently enough to use them effectively (and over the long term) with the minimal training and monitoring provided. For example, on the postintervention questionnaires, providers reported being able to use "eliciting self motivational statements", a critical MI strategy, with only half of the clients whom they were able to contact and counsel.

While all sites had plans for ongoing training that included additional practice and "coaching" sessions, on-site monitoring and regularly scheduled case review meetings, organisational and budgetary factors often prevented these plans from being implemented to the desired extent. Had providers' time been more flexible, a trainer or investigator at each site would have met with study providers on a routine basis, perhaps weekly in the beginning, and then monthly as providers became more skilled, to discuss study cases. The training team had also intended to conduct more ongoing practice of role play scenarios to test out new strategies for enhancing client participation and overcoming challenges. Since the study period was limited and we were working with existing agency personnel, the trainers were unable to require a certain level of proficiency before delivering the intervention. Instead, trainers did their best to help all providers incorporate the basic style of MI through additional practice role plays.

Our suggestion is that future studies looking to enhance behaviour change in health care settings allocate sufficient funding to instill and maintain quality training and monitoring of the intervention throughout the study period. This may also include the ability to "purchase" release time for providers so that client coverage does not interfere with provider skills development and monitoring of treatment fidelity.

\section{ORGANISATIONAL AND OTHER CONTEXTUAL}

FACTORS

In each of the organisations, providers were under pressure to handle large caseloads. Since none of the providers were employed specifically by the studies, they faced many competing demands. In most cases, providers saw a large number of patients each day and juggled diverse responsibilities. It is likely that given the busy workloads of the providers and the complexity of their patients' needs, the SFF study protocols were not always a priority. Ongoing changes in health care reimbursement within the organisation also added to the increasing demands placed upon providers to do more with fewer resources.

COMPETING PRIORITIES IN PROVIDERS'

PERCEPTIONS OF RISK FACTORS

It is difficult for providers to focus on one risk factor in "hard-to-reach" populations who typically have multiple and competing health and social support needs. Providers do not always see smoking, in particular, as a pressing issue. For example, in the pretraining questionnaires, providers at all sites rated other issues such as multiple substance abuse, poor nutrition, psychosocial issues, and stress as equal or higher than smoking in terms of health risks to patients. Providers' perception of the relative risks and importance of addressing smoking in this population may have interfered with their ability to most effectively encourage and help their patients make changes in their smoking. Although providers were encouraged to apply the motivational skills they learned to other health and psychosocial behaviours, limited time and access to their patients might have diluted their potential impact on their patients' smoking.

CONCLUSION

Additional time and ongoing resources need to be allocated to training health care providers to deliver motivational interventions in order to maximise their effectiveness. Health care organisations can provide additional support by identifying a team of providers who can serve as health behaviour counsellors. Providers who are interested and committed to learning new motivational skills to enhance health behaviour change can attend basic and ongoing training workshops to provide them with enhanced skills to address patients' health concerns. Following training workshops, trainers can assist organisations in identifying those individuals who may be best suited to deliver motivational interventions. Caseload assignment must be adjusted to allow providers sufficient time to interact with their patients while helping them work through the process of change. Funding agencies supporting trials of motivational interventions should plan to allocate sufficient funding for trainers to provide ongoing training and monitoring of intervention delivery to ensure that the intervention being studied is conducted at the highest quality level.

This research was supported by the Robert Wood Johnson Foundation, Smoke-Free Families Initiative The authors wish to acknowledge the intervention providers at Dana-Farber, Kaiser Permanente, and the University of Texas.

1 Rollnick S, Heather N, Bell A. Negotiating behavior change in medical settings: the development of brief motivational in medical settings: the development of brief motivation

2 Rollnick S, Mason P, Butler C. Health behavior change. University of Wales, Cardiff, UK: Churchill Livingstone, 1999

3 Rollnick S, Miller WR. What is motivational interviewing? Behavioral and Cognitive Psychotherapy 1995;23:325-34

4 Heather N, Rollnick S, Bell A, Richmond R. Effects of brief counselling among heavy drinkers identified on general hospital wards. Drug \& Alcohol Review 1996;15:29-38.

5 Butler CC, Rollnick S, Cohen D, Bachman M, Russell I, Stott N. Motivational consulting versus brief advice for smokers in general practice: a randomized trial. $\mathrm{Br} \mathcal{F} \mathrm{Gen}$ Pract 1999;49:611-16.

6 Miller WR, Rollnick S. Motivational interviewing: preparing people to change addictive behaviors. New York: Guilford Press, 1991.

7 Emmons KM, Sorensen G, Klar N, et al. Healthy baby second-hand smoke study: project brief. Tobacco Control 2000;9(suppl III):iii58-9.

8 Dolan-Mullen P, DiClemente CC, Velasquez MM, et al. Enhanced prenatal case management for low income smokers. Tobacco Control 2000;9(suppl III):75-7.

9 Ershoff DH, Quinn VP, Boyd NR, Stern J, Gregory M, Wirtschafter D. The Kaiser Permanente prenatal smokingcessation trial: when more isn't better, what is enough? Am F Prev Med 1996;17:161-68. 